\title{
ASCO-update: gastrointestinal tumors
}

\author{
Lukas Weiss (D) F Florian Huemer · Richard Greil
}

Received: 10 August 2017 / Accepted: 2 November 2017 / Published online: 13 November 2017 (C) The Author(s) 2017. This article is an open access publication.

Summary A multitude of new molecular and immunologic insights into gastrointestinal tumors have been presented at the ASCO meeting 2017; however, we have focused our update on practice-changing phase 3 trials and tried to set them into clinical perspective. Furthermore, we will elaborate on updated data on immunotherapeutics in gastroesophageal cancers, since drug approvals may be anticipated before convening for the next meeting in 2018 .

Keywords Cololrectal cancer · Gastric cancer · Biliary tract cancer $\cdot$ Immune checkpoint inhibitor

\section{Risk-adapted duration of adjuvant chemotherapy in stage III colon cancer}

In stage III colon cancer, adjuvant chemotherapy using FOLFOX (5-Fluorouracil, Leucovorin, Oxaliplatin) or CAPOX (Capecitabine, Oxaliplatin) is well established due to its documented prolongation of disease free $[1,2]$ as well as overall survival [3].

However, oxaliplatin-induced peripheral neurotoxicity poses a relevant clinical problem affecting more than $90 \%$ of patients when treated with 6 months of FOLFOX and more than $10 \%$ with grade 3 or 4 . Since oxaliplatin is associated with cumulative, dose-dependent toxicity, it is highly relevant to determine whether the duration of oxaliplatin-based chemotherapy (FOLFOX or CAPOX) can be shortened without losing clinical efficacy.

\footnotetext{
L. Weiss, MD, PhD (ه)

IIIrd Medical Department of Hematology, Medical Oncology, Hemostaseology, Rheumatology and Infectious Disease, Salzburg Cancer Research Institute (SCRI), Paracelsus Medical University, Muellner Hauptstraße 48, 5020 Salzburg, Austria

lu.weiss@salk.at
}

The IDEA (International Duration Evaluation of Adjuvant Chemotherapy) collaboration [4] pooled data from six phase III trials conducted in 12 countries and included more than 12,000 patients (see Table 1). These trials investigated the non-inferiority of 3 versus 6 months of adjuvant treatment with the primary endpoint of disease-free survival (DFS).

Patient characteristics were well balanced between the two treatment arms.

The estimated 3-year DFS in patients receiving 3 months of adjuvant chemotherapy (74.6\%) was lower than that in patients receiving 6 months of adjuvant chemotherapy $(75.5 \%)$ by $0.9 \%$ (HR 1.07 , $95 \%$ CI $[1.00,1.15])$. Since the upper limit of the CI exceeded the predefined non-inferiority margin of 1.12 for the DFS hazard ratio, non-inferiority was not established.

Despite this formally negative trial, preplanned subgroup analyses have possibly led to practice changing results:

Patients with lower risk disease defined as T1-3 $\mathrm{N} 1$ represented roughly $60 \%$ of patients. In this subgroup, non-inferiority of 3 months therapy could be established when compared to 6 months therapy with a DFS HR of 1.01 (95\% CI 0.90-1.12).

However, this was not the case for patients with higher risk disease defined as T4 or N2 (DFS HR 1.12, 95\% CI 1.03-1.23), favoring 6 months over 3 months of adjuvant therapy.

In a not prespecified combined DFS comparison by risk group and regimen, true non-inferiority in lower risk patients was only found for CAPOX (DFS HR 0.85, 95\% CI 0.71-1.01), but not for FOLFOX (DFS HR 1.10, 95\% CI 0.96-1.26).

The investigators hypothesized that possible reasons for a superiority in CAPOX over FOLFOX may either lie in the higher oxaliplatin dose received in the first 4 weeks of treatment (CAPOX $260 \mathrm{mg} / \mathrm{m}^{2}$; FOL- 
Table 1 IDEA trials

\begin{tabular}{l|l|l|l|l|}
\hline Trial & Regimen(s) & Stage & Tumor location & CAPOX (\%) \\
\hline TOSCA & CAPOX or FOLFOX4 & II \& III & Colon & 35 \\
\hline SCOT & CAPOX or mFOLFOX6 & II \& III & Colon \& rectum & 67 \\
\hline IDEA France & CAPOX or mFOLFOX6 & III & Colon & 10 \\
\hline C80702 & mFOLFOX6 & III & Colon & 0 \\
\hline HORG & CAPOX or FOLFOX4 & II \& III & Colon & 58 \\
\hline ACHIEVE & CAPOX or mFOLFOX6 & III & Colon & 76 \\
\hline FOLFOX 5-Fluorouracil, Leucovorin, OXaliplatin, CAPOX Capecitabine, Oxaliplatin & &
\end{tabular}

FOX $170 \mathrm{mg} / \mathrm{m}^{2}$ ) or in the more continuous exposure to capecitabine in the CAPOX regimen when compared to 5-FU in the FOLFOX regimen.

The goal of lowering burdensome neurotoxicity by shortening treatment duration was achieved: patients receiving 3 months of treatment had significantly lower rates of grade $\geq 2$ neurotoxicity (3 months vs 6 months: $17 \%$ vs $48 \%$ [FOLFOX] and $15 \%$ vs $45 \%$ [CAPOX]; $P=0.0001$ ). Presumably as a consequence thereof, compliance was worse in the 6-month arm than in the 3-month arm, irrespective of the regimen used (3 months vs 6 months: 90\% vs. 71\% [FOLFOX]; 3 months vs 6 months: $86 \%$ vs $65 \%$ [CAPOX]).

Take home message Based on these results of the IDEA trials, the authors recommend a risk-adapted approach to selecting adjuvant chemotherapy duration for stage III colon cancer:

- T1-3, N1: 3 months of CAPOX or FOLFOX

- T4 or N2: 6 months of FOLFOX

Although patients with stage III rectal cancer were only included in the SCOT trial (without preoperative chemoradiotherapy), it might be appropriate to also extrapolate the above mentioned recommendations to rectal cancer.

\section{Adjuvant capecitabine for biliary tract cancers}

The BILCAP trial examined adjuvant capecitabine in patients with resected biliary tract cancers [5]: in this two-arm, open-label, controlled study, 447 patients were randomly assigned to observation or to capecitabine $\left(1250 \mathrm{mg} / \mathrm{m}^{2}\right)$ twice daily for 8 cycles.

In the ITT population, capecitabine was associated with a median OS of 51.1 months (95\% CI [34.6, 59.1]), compared to 36.4 months with observation $(95 \% \mathrm{CI}$ $[29.7,44.5])$. Despite this 15-month difference statistical significance was not reached (HR 0.81, 95\% CI $[0.63,1.04] ; p=0.097)$, which was however reached in the per-protocol population (HR 0.75, 95\% CI [0.58, 0.97 ]; $p=0.028$ ), with a median OS of 52.7 months in the capecitabine group compared to 36.1 months in the observation group.

Similarly significant improvements were also seen in relapse-free survival (RFS): 24.6 months in the capecitabine arm vs 17.6 months in the observation arm (HR 0.76, $P=0.039$ ). Adverse events were well manageable with palmar-plantar erythrodysesthesia as the most common grade $3 / 4$ adverse event (21\%).

Take home message In the light of the significantly prolonged RFS, adjuvant capecitabine should become the standard of care in resected biliary tract cancers and should be used as control arm in future adjuvant trials. With great interest we are awaiting the results of the currently ongoing adjuvant ACTICCA-1 trial (NCT02170090) using gemcitabine and cisplatin which will challenge the role of adjuvant capecitabine.

\section{Perioperative FLOT for resectable gastric and GEJ cancer}

The FLOT4 trial [6] compared perioperative chemotherapy with FLOT versus ECF/ECX in patients with resectable gastric cancer or adenocarcinoma of the gastroesophageal junction (types I to III; cT2-T4 or cT any and $\mathrm{cN}+$ ). In this multicenter, randomized Phase 3 trial 716 patients were randomly assigned to receiving 4 preoperative and 4 postoperative cycles of FLOT every 2 weeks (docetaxel $50 \mathrm{mg} / \mathrm{m}^{2}$, day 1; 5-FU $2600 \mathrm{mg} / \mathrm{m}^{2}$ over $24 \mathrm{~h}$, day 1 , leucovorin $200 \mathrm{mg} / \mathrm{m}^{2}$, day 1; oxaliplatin $85 \mathrm{mg} / \mathrm{m}^{2}$, day 1) versus receiving 3 preoperative and 3 postoperative cycles of ECF/ECX (epirubicin, cisplatin, 5-FU or capecitabine).

The primary endpoint OS could be significantly improved with FLOT (median 50 months) when compared to ECF/ECX with a median OS of 35 months (HR 0.77, $P=0.012$ ). Accordingly, PFS improved by 12 months in patients receiving FLOT (30 months vs 18 months; HR $0.75, P=0.004$ ). This effect could be consistently observed across histological and clinical subgroups.

Furthermore, FLOT resulted in a higher rate of pathologic remissions with $25 \%$ of $\leq$ ypT1 versus $15 \%$ with ECF/ECX $(P=0.001)$.

Rates of serious adverse events were similar between the two treatment arms and perioperative FLOT did not increase surgical morbidity or mortality, the rate of resurgeries or hospitalization times when compared to ECF/ECX.

Take home message The final results of the FLOT4 phase 3 trial have been long awaited and now con- 
firm the results of the previous phase 2 and a clinical practice which has already been adopted in many European centers. Therefore, FLOT can be considered the new standard of perioperative chemotherapy for resectable gastric or gastroesophageal adenocarcinoma.

In light of the fact that only half of the patients have completed their allocated postoperative chemotherapy, future clinical trials are aiming at the intensification of preoperative therapy or at employing new types of therapy in the postoperative setting such as immune checkpoint inhibitors.

The role of immune checkpoint inhibitors in gastroesophageal cancers - updated and confirmed

Especially important from a European perspective: two trials mainly including patients from western countries have been updated at ASCO this year and could confirm the promising results observed in previous Asian trials:

The cohort 1 of the phase 2 trial KEYNOTE-059 has included 259 patients that have previously been treated with 2 or more lines of chemotherapy [7]. Patients were mainly recruited form the US (47.9\%), only $13.1 \%$ from East Asia and $39.0 \%$ from the rest of the world.

PDL-1 expression was positive in $57.1 \%$ of patients (defined as a combined positive score of $\geq 1 \%$ ) and although PD-Ll positivity was associated with a higher overall response rate (ORR) of $15.5 \%, \mathrm{PD}-\mathrm{L} 1$ negativity did not preclude patients from response (ORR $6.4 \%$ ). Indeed, similar rates of complete responses were seen in the PD-L1 positive $(2.0 \%)$ and the PDL1 negative cohort (2.8\%). However, a certain decline in ORR could be observed with an increasing number of previous lines of therapy (3 lines: 16.4\%; 4 lines: $6.4 \%$ ). Best responses were seen in patients with MSI high tumors, where a response was seen in 4 out of 7 patients.

Overall, treatment with pembrolizumab $(200 \mathrm{mg}$ every 3 weeks) resulted in an ORR of $11.6 \%$ and the reduction in tumor in $42.4 \%$ of patients.

The phase 1/2 CheckMate 032 trial compared the combination of nivolumab and ipilimumab to nivolumab monotherapy in 160 patients with advanced/metastatic gastric or gastroesophageal cancer [8].

Two different schedules of the combination therapy were compared to standard doses nivolumab $(3 \mathrm{mg} / \mathrm{kg}$ IV every 2 weeks). Patients receiving the combination nivolumab $(1 \mathrm{mg} / \mathrm{kg})$ and ipilimumab $(3 \mathrm{mg} / \mathrm{kg}) \mathrm{had}$ an ORR of $24 \%$, compared with $12 \%$ in patients receiving nivolumab alone. Of interest, ORR in the combinations arms seemed to be dependent on dosing, as only $8 \%$ patients who received the alternate dosing (nivolumab $3 \mathrm{mg} / \mathrm{kg}$ and ipilimumab $1 \mathrm{mg} / \mathrm{kg}$ ) re- sponded. Responses were observed regardless of PDL1 expression.

As expected, combination therapy was associated with higher serious toxicity (NIVO 1 \& IPI 3: $43 \%$; NIVO: $10 \%$ ).

Take home message The corroborated positive results obtained with immune checkpoint inhibitors in gastroesophageal cancers will pave the way for their implementation in the clinics following the anticipated approvals of pembrolizumab and nivolumab in 2018.

Funding Open access funding provided by Paracelsus Medical University.

Conflict of interest L. Weiss, F. Huemer, and R. Greil declare that they have no competing interests.

Open Access This article is distributed under the terms of the Creative Commons Attribution 4.0 International License (http://creativecommons.org/licenses/by/4.0/), which permits unrestricted use, distribution, and reproduction in any medium, provided you give appropriate credit to the original author(s) and the source, provide a link to the Creative Commons license, and indicate if changes were made.

\section{References}

1. AndreT, BoniC, Mounedji-BoudiafL, Navarro M, Tabernero J, Hickish T, et al. Oxaliplatin, fluorouracil, and leucovorin as adjuvant treatment for colon cancer. N Engl J Med. 2004;350(23):2343-51.

2. Haller DG, Tabernero J, Maroun J, de Braud F, Price T, Van Cutsem E, et al. Capecitabine plus oxaliplatin compared with fluorouracil and folinic acid as adjuvant therapy for stage III colon cancer. JClin Oncol. 2011;29(11):1465-71.

3. Andre T, Boni C, Navarro M, Tabernero J, Hickish T, Topham C, et al. Improved overall survival with oxaliplatin, fluorouracil, and leucovorin as adjuvant treatment in stage II or III colon cancer in the MOSAIC trial. J Clin Oncol. 2009;27(19):3109-16.

4. Shi Q, Sobrero AF, Shields AF, Yoshino T, Paul J, Taieb J, et al. Prospective pooled analysis of six phase III trials investigating duration of adjuvant (adjuv) oxaliplatin-based therapy (3 vs 6 months) for patients (pts) with stage III colon cancer (CC): The IDEA (International Duration Evaluation of Adjuvant chemotherapy) collaboration. J Clin Oncol. 2017;35(18_suppl):LBAl-LBA.

5. Primrose JN, Fox R, Palmer DH, Prasad R, Mirza D, Anthoney DA, et al. Adjuvant capecitabine for biliary tract cancer: the BILCAP randomized study. J Clin Oncol. 2017;35(15_suppl):4006.

6. Al-Batran S-E, Homann N, Schmalenberg H, Kopp H-G, Haag GM, Luley KB, et al. Perioperative chemotherapy with docetaxel, oxaliplatin, and fluorouracil/leucovorin (FLOT) versus epirubicin, cisplatin, and fluorouracil or capecitabine (ECF/ECX) for resectable gastric or gastroesophageal junction (GEJ) adenocarcinoma (FLOT4-AIO): a multicenter, randomized phase 3 trial. J Clin Oncol. 2017;35(15_suppl):4004.

7. Fuchs CS, Doi T, Jang RW-J, Muro K, Satoh T, Machado $\mathrm{M}$, et al. KEYNOTE-059 cohort 1: efficacy and safety of pembrolizumab (pembro) monotherapy in patients with 
previously treated advanced gastric cancer. J Clin Oncol. 2017;35(15_suppl):4003.

8. Janjigian YY, Ott PA, Calvo E, Kim JW, Ascierto PA, Sharma $\mathrm{P}$, et al. Nivolumab \pm ipilimumab in pts with advanced (adv)/metastatic chemotherapy-refractory (CTx-R) gastric $(\mathrm{G})$, esophageal $(\mathrm{E})$, or gastroesophageal junction (GEJ) cancer: CheckMate 032 study. J Clin Oncol. 2017;35(15_suppl):4014.

- For latest news from international oncology congresses see: http://www.springermedizin.at/ memo-inoncology 